\title{
Electrophysiologic Recurrent Laryngeal Nerve Monitoring During Thyroid and Parathyroid Surgery: International Standards Guideline Statement
}

Gregory W. Randolph, MD; Henning Dralle, MD, with the International Intraoperative Monitoring Study Group*: Hisham Abdullah, MD; Marcin Barczynski, MD; Rocco Bellantone, MD; Michael Brauckhoff, MD;

Bruno Carnaille, MD; Sergii Cherenko, MD; Fen-Yu Chiang, MD; Gianlorenzo Dionigi, MD, FACS;

Camille Finck, MD; Dana Hartl, MD; Dipti Kamani, MD; Kerstin Lorenz, MD; Paolo Miccolli, MD;

Radu Mihai, MD, PhD, FRCS; Akira Miyauchi, MD, PhD; Lisa Orloff, MD, FACS; Nancy Perrier, MD, FACS;

Manuel Duran Poveda, MD; Anatoly Romanchishen, MD; Jonathan Serpell, MD, FRACS, FACS;

Antonio Sitges-Serra, MD; Tod Sloan, MD, MBA, PhD; Sam Van Slycke, MD; Samuel Snyder, MD, FACS;

Hiroshi Takami, MD; Erivelto Volpi, MD; Gayle Woodson, MD

Intraoperative neural monitoring (IONM) during thyroid and parathyroid surgery has gained widespread acceptance as an adjunct to the gold standard of visual nerve identification. Despite the increasing use of IONM, review of the literature and clinical experience confirms there is little uniformity in application of and results from nerve monitoring across different centers. We provide a review of the literature and cumulative experience of the multidisciplinary International Neural Monitoring Study Group with IONM spanning nearly 15 years. The study group focused its initial work on formulation of standards in IONM as it relates to important areas: 1) standards of equipment setup/endotracheal tube placement and 2) standards of loss of signal evaluation/intraoperative problemsolving algorithm. The use of standardized methods and reporting will provide greater uniformity in application of IONM. In addition, this report clarifies the limitations of IONM and helps identify areas where additional research is necessary. This guideline is, at its forefront, quality driven; it is intended to improve the quality of neural monitoring, to translate the best available evidence into clinical practice to promote best practices. We hope this work will minimize inappropriate variations in monitoring rather than to dictate practice options.

Key Words: Recurrent laryngeal nerve, nerve monitoring, intraoperative neural monitoring, international standards, guidelines for intraoperative neural monitoring, thyroid surgery, parathyroid surgery, nerve injury, nerve monitoring equipment, neural mapping, nerve identification, anesthesia and nerve monitoring, loss of signal, laryngeal twitch, vagus nerve, electromyography characteristics, vocal cord mobility, latency, amplitude, superior laryngeal nerve.

Level of Evidence: 5 .

Laryngoscope, 121:S1-S16, 2011

From the Department of Otology and Laryngology, Division of Thyroid and Parathyroid Surgery, Massachusetts Eye and Ear Infirmary, Harvard Medical School, (G.w.R.), Division of Surgical Oncology, Endocrine Surgery Service, Department of Surgery, Massachusetts General Hospital, Harvard Medical School, (G.W.R.), Boston, Massachusetts, U.S.A., Department of Surgery and Department of General, Visceral, and Vascular Surgery, University of Halle, Halle, Germany (H.D.). $14,2010$.

Editor's Note: This Manuscript was accepted for publication June

The authors have no funding, financial relationships, or conflicts of interest to disclose.

*Drs. Randolph and Dralle are members of the International Intraoperative Monitoring Study Group.

Send correspondence to Gregory W. Randolph, Department of Otolaryngology, Division of Thyroid and Parathyroid Surgery, Massachusetts Eye and Ear Infirmary, 243 Charles St., Boston, MA 02114

E-mail: Gregory_Randolph@meei.harvard.edu

DOI: 10.1002/lary.21119

\section{INTRODUCTION/RATIONALE}

The purpose of this report is to provide a review of the clinical experience of the International Neural Monitoring Study Group with respect to standards in electrophysiologic intraoperative neural monitoring (IONM) during thyroid and parathyroid surgery. The International Neural Monitoring Study Group is a multidisciplinary international group of surgeons and researchers selected based on clinical experience and expertise in thyroid and parathyroid surgery, neural monitoring, and related fields. This group includes surgeons (including otolaryngologists and general surgeons), laryngologists, voice and laryngeal electromyography (EMG) specialists, and anesthesiologists. IONM is a multistep process with a complex set of equipment challenges. This guideline is presented as an initial 
construct focused primarily on standardization of IONM as it relates to important areas within IONM: standards of equipment setup/endotracheal tube placement and standards of loss of signal (LOS) evaluation/intraoperative problem-solving algorithm.

This guideline development attempt is primarily quality driven; it is intended to improve the quality of neural monitoring and to reduce inappropriate variations in monitoring technique. Through this work we sought to reduce the level of uncertainty in IONM by clarifying the limitations of knowledge about IONM and to help identify those areas where additional research is necessary. The use of standardized methods and reporting will support future studies exploring the full utility of IONM, including key studies correlating intraoperative electrophysiologic data with postoperative glottic function.

The study group's attempt in guideline formation was constrained by the available data. We reviewed the evidence-based literature and the cumulative experience of the multidisciplinary study group with IONM spanning nearly 15 years. The two senior authors combined have experience with neural monitoring starting in 1993 of approximately 8,000 cases. The guideline evolved through several iterations and was the result of group consensus. The International Neural Monitoring Study Group was formed as a working group by and is associated with the European Society of Endocrine Surgery and has met formally once a year from 2006. This large, multidisciplinary study group has diverse expertise and perspective, which helps minimize bias. The study group makes its recommendations based solely on the literature and the group's cumulative clinical surgical experience. This report does not endorse any specific company or set of monitoring equipment.

IONM during thyroid and parathyroid surgery has gained widespread acceptance as an adjunct to the gold standard of visual nerve identification, adding a new functional dynamic during thyroid surgery. Rates of monitoring use have recently become more or less equivalent between general surgical and otolaryngology-trained surgeons, with approximately $40 \%$ to $45 \%$ in both groups using IONM in some or all cases. ${ }^{1,2}$ Within the United States, monitoring appears to be used by younger surgeons and surgeons with more than 100 cases per year. ${ }^{2}$ Thus it appears that, at least within the United States, monitoring has been found to have utility by not only the younger novice but also by the high-volume experienced thyroid surgeon who is completely familiar with thyroid and recurrent laryngeal nerve (RLN) anatomy. Despite this increasingly broad use of IONM, review of the literature and clinical experience of the study group confirms there is little uniformity in nerve monitoring across different centers. For example, laryngeal exam may not be performed preoperatively and postoperatively, a variety of different recording electrodes may be used (including vocal cord surface electrodes, vocal cord needle electrodes, and postcricoid paddle electrodes), and a variety of different stimulation electrodes may be used (including both monopolar and bipolar neural stimulators). Monitoring systems also vary, with some depicting the laryngeal EMG waveform and others providing only an audio tone (based on EMG interpretation); still others combine both the visual display of the waveform and audio tones. Further, there are no standard algorithms for endotracheal tube placement, loss of EMG signal troubleshooting system evaluation, or even the basic modes of IONM application.

A recent evidence-based literature review of nonrandomized studies looking at rates of nerve paralysis with and without monitoring with more than 100 nerves at risk showed divergent results. ${ }^{3-18}$ It is of note that recent randomized work of Barczyński demonstrated statistically lower rates for transient paralysis (lower by $2.9 \%$ in high-risk group) with neural monitoring as compared to visual identification alone. ${ }^{19}$ Dralle has studied issues of statistical power necessary to prove that rates of paralysis are lower with the application of neural monitoring. His studies have suggested that a researcher would need 9 million patients per arm for benign multinodular goiter and approximately 40,000 patients per arm for thyroid cancer for such studies to be conducted with statistical power if typical rates of nerve paralysis are used for calculation. ${ }^{16}$

The study group has defined three discrete modes of IONM application:

1. Identification (neural mapping) of the RLN. The nerve is mapped out in the paratracheal region through stimulation and then visually identified through directed dissection provided by the neural mapping. Multiple studies suggest IONM is associated with rates of nerve identification between $98 \%$ and $100 \% .^{20}$

2. Aid in dissection. Once the nerve is identified, additional intermittent stimulation of adjacent nonneural tissue versus nerve can help in tracing the nerve and all its branches through the dissected field in a way analogous to the use of intermittent facial nerve stimulation as one dissects the facial nerve during parotidectomy.

3. Prognostication of postoperative neural function and lesion site identification. This application has great significance in prevention of bilateral vocal cord paralysis given the bilateral nature of the typical thyroid procedure. Prognostic statistics ${ }^{21}$ vary owing to a number of factors discussed in this report, but electric testing represents a significant improvement in accuracy of neural-function prognostic testing when compared to the currently available test of visual inspection of the nerve. Further, before neural testing there has been no mechanism to identify the segment of nerve injured.

There are reports in the literature suggesting significant inaccuracies from nonstandard application of monitoring techniques. ${ }^{3,5-16,18,21-25}$ Novice monitoring surgeons underuse vagal stimulation during IONM (Dionigi, personal communication, 2010). Existing studies have shown postoperative neural function prediction with IONM is associated with uniform and high negative predictive values ranging from $92 \%$ to $100 \% .{ }^{26}$ However, studies primarily using audio-only systems have shown 
positive predictive values that are generally quite low and highly variable, ranging from $9.2 \%$ to 92.1\%. ${ }^{6,16,19,21,26}$ Based on existing prognostic studies, uniform and robust loss of signal (LOS) evaluation algorithms would be expected to increase and provide more uniform positive predictive values. Study group members felt that it was important that standards be applied to IONM especially as it relates to equipment setup and to system assessment/troubleshooting to facilitate uniform comparable and accurate neural monitoring.

The study group believes that neural monitoring could be performed routinely given that difficult cases cannot always be predicted preoperatively. Even if nerve monitoring yields the greatest advantage in difficult thyroid operations, routine application has shown to steepen learning curves through greater experience in interpretation of the signal and troubleshooting system malfunction. ${ }^{24}$ It is of note that within the literature and reviewing the cumulative experiences of the international study group members, repetitive stimulation of the RLN or vagus nerve is not associated with neural injury and has been applied safely in children and adults. ${ }^{27}$ Further vagal stimulation is unassociated with brady arrhythmias or bronchospasm. ${ }^{28}$

The study group believes there are certain minimum or overarching essential elements for optimal IONM that include the following:

1. Preoperative laryngoscopy is necessary in all cases. With neural monitoring intraoperatively we are assessing the functional integrity of the RLNs bilaterally. It is therefore essential that we are aware of the functional status of the vocal cords before the beginning of surgery through preoperative glottic exam. The need for accurate preoperative glottic function information in all cases is essential for accurate monitoring and for other reasons and has been detailed elsewhere. ${ }^{29,30}$

2. Presurgical dissection suprathreshold vagal nerve stimulation allows for verification of IONM system function and therefore allows for subsequent neural mapping for the RLN with accuracy (i.e., a negative stimulation can be relied on as a true negative).

3. Postsurgical dissection suprathreshold vagal stimulation allows for the most accurate prognostication testing of postoperative glottis function. Dralle et al. have shown higher sensitivity, slightly higher specificity, higher positive predictive value, and slightly higher negative predictive value for vagal stimulation as opposed to RLN stimulation in the prediction of vocal cord paralysis postoperatively. ${ }^{16}$ Vagal stimulation allows for testing of the entire neural circuit and avoids the potential false-negative scenario of stimulating a damaged RLN distal to the site of injury. It is important to note that with vagal stimulation, although there is great similarity with RLN stimulation, the vagus nerve is of larger caliber and the laryngeal motor fibers may be eccentrically placed. This means that a given stimulation of the vagus nerve may have amplitude that is somewhat smaller than RLN stimulation at a given stimulating current.
4. Postoperative laryngoscopy is necessary in all cases. Neural stimulation at the end of surgery and postoperative glottic function are highly correlated, but our understanding of this correlation has yet to be perfected. Postoperative laryngeal exam is essential in all cases while IONM is in the development phase to improve the prognostic correlation between end-of-surgery neural stimulation and postoperative glottic function. Accurate information about postoperative glottic function in all cases is essential for accurate monitoring as well as for other reasons and has been detailed elsewhere. ${ }^{29,30}$

\section{STANDARDS OF EQUIPMENT}

Many different nerve-monitoring formats have been studied, including laryngeal palpation, ${ }^{4,5,31,32}$ glottic observation, ${ }^{33-35}$ glottic pressure monitoring, ${ }^{36}$ endoscopically placed intramuscular vocal cord electrodes, ${ }^{37-39}$ intramuscular electrodes placed through the cricothyroid membrane, ${ }^{7,8,13,40,41}$ endotracheal tube-based surface electrodes, ${ }^{11,42-48}$ and postcricoid surface electrodes. ${ }^{49,50}$ For a variety of reasons including safety, utility, and simplicity, systems that rely on endotracheal tube-based surface electrodes have proliferated and represent the most common monitoring equipment format to date.

Current neural monitoring equipment can be broadly divided into audio-only systems and systems that provide both audio and visual waveform information regarding evoked waveform. Audio-only systems provide substantially less information such as waveform morphology, amplitude, threshold, and latency that may provide for basic understanding of amplitude variation in normal and pathologic conditions; this information may be important in surgical deliberations. Exact determination of LOS as well as differentiation between signal and artifact may be challenging if not impossible with audio-only systems. Audio-only systems are problematic in that the EMG response to RLN stimulation cannot be quantified. Response quantification is one of the real opportunities in EMG assessment. There are also difficulties in such systems with documentation of response. Another type of monitoring system involves visualization of the larynx during stimulation of the RLN. Such glottic visualization monitoring schemes have been inadequately studied. It can be difficult for even trained personnel to definitively diagnose clear-cut vocal cord movement, to quantify this movement, and to differentiate the movement as to abduction versus adduction. Notably, the basic relationship between neural stimulation during surgery and intraoperative vocal cord motion inspection as it relates to postoperative volitional function has been inadequately studied. It may well be that nonphysiologic stimulation of the RLN at surgery may result in some sort of evoked glottic motion, yet postoperatively volitional function is abnormal. For these reasons the study group believes endotracheal tube-based systems that include graphic monitor documentation of waveform are preferred for neural monitoring.

Recording electrodes are typically either needlebased or endotracheal tube-based but may include other 
surface arrays such as postcricoid electrodes. Needle electrodes do typically result in larger amplitude measures but overall offer no real advantage as opposed to the surface electrodes. ${ }^{51,52}$ Surface electrode measures are well correlated to needle electrode measures. ${ }^{28}$ Needle recording electrodes do raise the possibility of trauma, including vocal cord or laryngeal hematoma, vocal cord laceration, infection, cuff deflation and need for reintubation, retained fractured needle segment, and accidental needle dislodgement during surgery. Needle recording electrodes also only monitor unilaterally and so must be repositioned for each side. One advantage of needle electrodes is that significant displacement can at least be identified easily at the time of surgery. Endotracheal tube-based systems record EMG data from the vocal cord (i.e., thyroarytenoid or vocalis muscle), and postcricoid electrodes have been used to monitor the posterior cricoarytenoid (PCA) muscle, the main abductor of the glottis. It appears that such postcricoid electrodes are equally sensitive to vocal cord electrodes but do require additional equipment beyond an endotracheal tube and so have not become widely popular. ${ }^{49,50}$

Stimulating electrodes may be monopolar or bipolar and may also be configured as dissecting instruments. Current flow through dissecting instruments has not been adequately studied, and so the exact stimulating current transmitted to the nerve through dissecting stimulating instruments is unknown. Bipolar stimulating electrodes may offer the potential advantage of greater sensitivity through focal nerve stimulation. There is insufficient data in the literature to comment as to which stimulating electrode type is preferable. Bipolar stimulating electrodes may have some utility if one is experiencing frequent or diffuse false-positive stimulation. One must be aware that if a bipolar array is used for nerve stimulation, the exact orientation of the positive (anode) and negative (cathode) stimulating electrodes as they are placed on the nerve is of extreme importance in efficient nerve stimulation. In addition, the bipolar probe may not be optimal for mapping of the nerve because the stimulation is more focal at the point of contact as compared with the monopolar probe, which provides more diffuse current spread, which may facilitate mapping of a larger area.

\section{STANDARDS IN ANESTHESIA}

\section{Anesthesia Protocols for Neural Monitoring}

An essential ingredient in successful RLN and vagal monitoring is partnership with the anesthesiologist. It is important to discuss monitoring with the anesthesiologist before the initiation of a neural monitoring program. It is best to discuss the need to have no muscle relaxation during the monitoring in advance so the anesthesiologist will have time to prepare before the first planned monitored case.

Little has been written on the subject of neural-monitoring anesthesia, despite the proliferation of complex neuromonitoring techniques. Anesthesia must be titrated to each patient to adjust for various comorbidities and allow for an adequate monitoring signal while keeping the patient adequately anesthetized. The most challenging anesthetics will be those when monitoring techniques are sensitive to inhalational agents (IH) and/or neuromuscular blocking agents (NMB). Some monitoring modalities such as auditory evoked brainstem response are insensitive to both; others such as EMG are sensitive to muscle relaxants only, and others such as somatosensory evoked potentials are sensitive to IH only. Some more complex monitoring modalities are sensitive to both $\mathrm{IH}$ and muscle relaxants such as transcranial motor evoked potentials.

When monitoring employs EMG data derived from stimulation of cranial nerves, such as the facial nerve (during lateral skull base, mastoid, or parotid surgery) or vagal nerve (during thyroid and parathyroid surgery), monitoring becomes sensitive to neuromuscular blockade. For some EMG techniques where the nervous system is stimulated, such as transcranial motor evoked potentials, partial systemic muscle relaxation may be acceptable. However, when monitoring is designed to be sensitive to mechanical stimulation of the nerves, muscle relaxants reduce the EMG amplitude and make monitoring less sensitive to impending neural injury. Similarly, NMB may also reduce amplitude of evoked responses. ${ }^{53}$ For these reasons it is best, after induction, to allow all neuromuscular blockade to wear off and avoid NMB throughout the rest of the case. If spontaneous and evoked muscle activity is the only modality being monitored, as is the case in RLN and vagal nerve monitoring, aside from avoiding NMB during monitoring, the anesthesiologist has the freedom to choose the most appropriate anesthetic for the patient as these anesthesia agents have very little effect on peripheral nerves and muscles. Typical anesthesia protocols would involve initial IH including isoflurane or desflurane with or without nitrous oxide. A total intravenous anesthesia technique may also be used; this may include propofol and opioids such as remifentanil, fentanyl, or sufentanil. These recommendations are similar for pediatric cases as well. Of note, propofol infusion syndrome has been observed in children and adults. ${ }^{54}$

It is essential to have full muscular activity return as soon as possible subsequent to intubation. Therefore, succinyl choline at 2 to $2.5 \mathrm{mg}$ per kilogram or a small dose of a nondepolarizing muscle relaxant (e.g., rocuronium and atracurium at $0.5 \mathrm{mg} / \mathrm{kg}$ ) may be used at intubation to allow for normal return of spontaneous respiration and resumption of normal muscle twitch activity within several minutes. It is important to keep in mind that a preoperatively unknown pseudocholinesterase deficiency will lead to prolonged paralysis after a depolarizing muscle relaxant such as succinyl choline and will invalidate an EMG monitoring system. ${ }^{12}$ It is well established that there is a significant difference in the degree of relaxation of the adductor pollicis muscle (this muscle being used typically to assess muscular activity and degree of neuromuscular blockade through transcutaneous stimulation) and the vocalis and other laryngeal muscles. The laryngeal muscles and diaphragm share a common and unique time course of paralysis with neuromuscular blockage relative to systemic skeletal muscle including the adductor pollicis. The larynx exhibits shorter response 

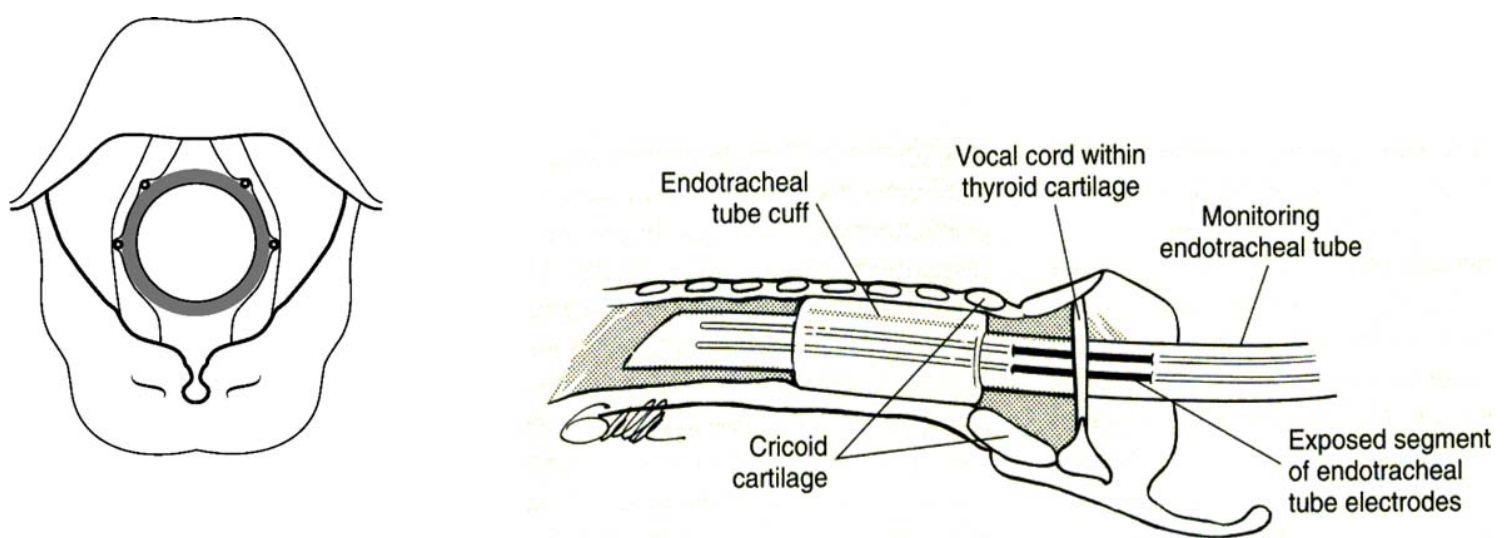

Fig. 1. Monitoring endotracheal tube in position. [Color figure can be viewed in the online issue, which is available at wileyonlinelibrary.com.]

time and recovers more quickly from neuromuscular blockade. ${ }^{55-58}$ Marusch et al. found that with NMB there was measurable EMG from the vocalis muscle but at systemic muscular relaxation degrees of $>90 \%$, vocalis muscle EMG amplitude became reduced. ${ }^{55}$ Although systemic paralytic agents can be given so as to effect systemic relaxation with less reduced neuromuscular blockage at the level of the larynx, every administration of a paralytic agent during the case has the potential for at least some degree of reduction in optimal laryngeal monitoring response. Any ongoing paralytic agent administration could attenuate EMG responses and would prevent quantitative analysis of the EMG data at the completion of surgery, making postoperative prognostic schemes less accurate.Given that all patients can be successfully cared for with general anesthetics without paralytic agents (after induction), the study group recommends strongly against the administration of any paralytic agents during a case in which monitoring is being employed if one desires optimal and quantifiable laryngeal response.

It is of note that nitrous oxide, other gas $\mathrm{IH}$, and intravenous narcotics do not affect EMG readings. The depth of anesthesia from these agents must be sufficient to avoid any spontaneous activity of the vocal cords. This level of anesthesia may be deeper than usually employed when neuromuscular blockage is used. If baseline EMG activity is substantially high because the plane of anesthesia is too light, it will be difficult to differentiate spontaneous activity from intentionally evoked (i.e., stimulated) activity. There has been some limited experience within the study group showing that sevoflurane as an IH is associated with higher baseline EMG activity at the level of the larynx than other IHs. This issue can be resolved by switching to isoflurane or desflurane (Sloan and Randolph, unpublished observations).

\section{STANDARDS OF EQUIPMENT SETUP/ ENDOTRACHEAL TUBE PLACEMENT}

\section{Monitoring Tubes}

Monitoring endotracheal tubes may be prefashioned with integrated paired left and right stainless steel electrodes embedded within the endotracheal tube surface that are exposed at the level of the glottis. Alternately, standard endotracheal tubes may be made into monitoring tubes by placement of a thin adhesive pad containing the paired electrodes. When attaching such electrodes, the lower tip of the electrode is generally placed approximately 7 to $10 \mathrm{~mm}$ above the upper edge of the endotracheal tube cuff. It is important that the adhesive pad electrode is placed and pressed firmly onto the endotracheal tube without any gaps and that the electrode does not overlap on itself, which sometimes occurs with smaller endotracheal tubes. Trimming the lateral edge of the electrode may be required. Whether a prefashioned endotracheal tube or adhesive pad electrode endotracheal tube is used, the endotracheal tube is designed to have the electrodes at the level of the glottis when the endotracheal cuff is in its normal position in the subglottis (Fig. 1 ). The endotracheal tube electrodes (referred to as the recording electrodes) when correctly placed will make contact with the medial surface of the bilateral cords to allow for monitoring of the bilateral thyroarytenoid/vocalis muscle's surface summated depolarization.

Intraoperative nerve monitoring involves multifaceted electronic recording and stimulation equipment. Use of this equipment introduces the potential for equipment-associated error at several discrete points in the monitoring system. A number of series have reported that significant equipment problems, mostly relating to the endotracheal tube, have been seen in $3.8 \%$ to $23 \%$ of monitored patients. ${ }^{12,22,59}$

Monitoring systems can, for problem-solving purposes, generally be divided into the following categories:

1. The recording side involves the endotracheal tube recording electrodes, its recording electrode ground, and associated connections at the interface-connector box and monitor.

2. The stimulation side includes the stimulation neural probe, its grounding electrode, and associated connections to the interface box-connector and stimulation current pulse generator within the monitor (Fig. 2).

With experience, one finds the majority of equipment-related problems are related to malpositioned endotracheal tube recording electrodes. The study group 


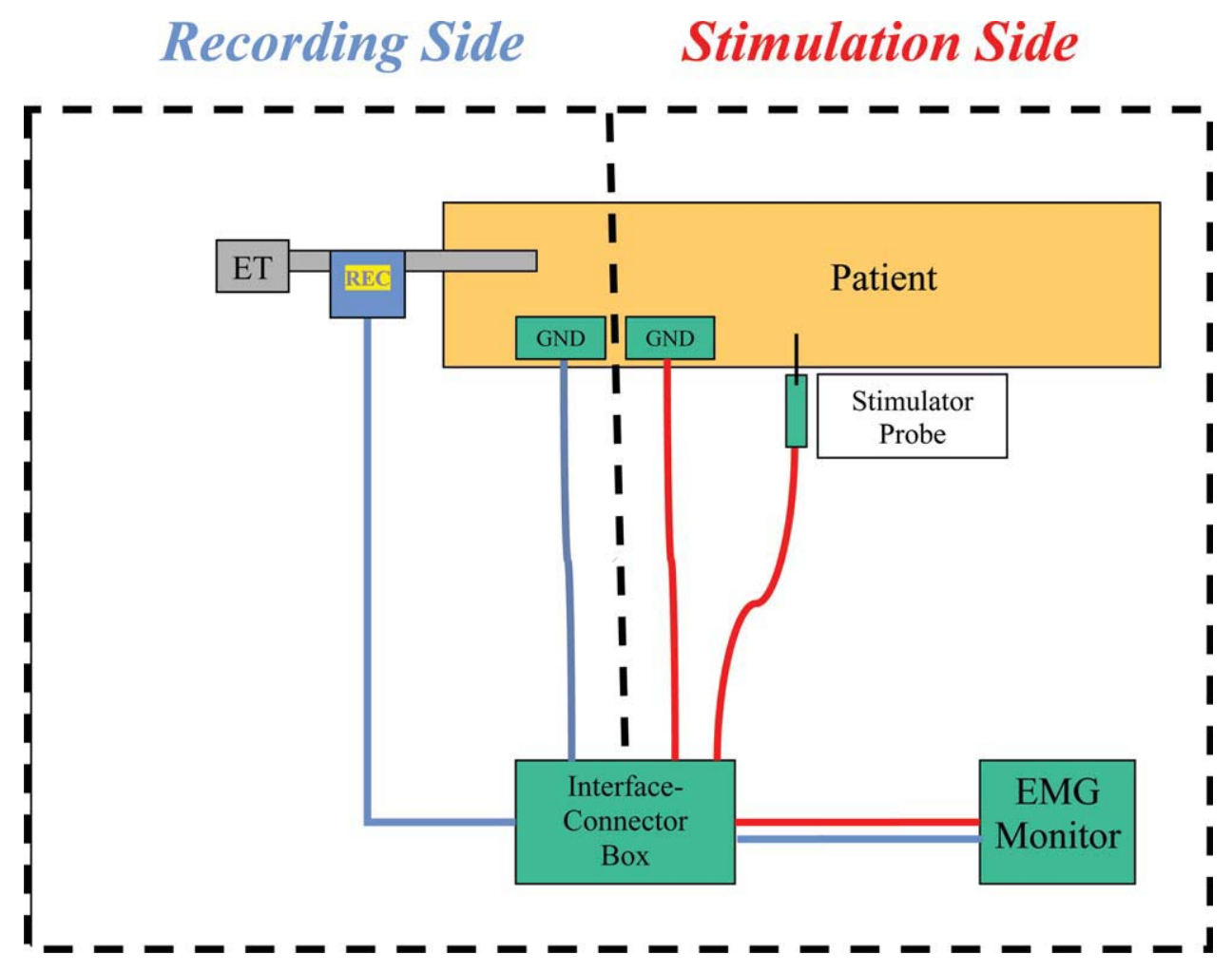

Fig. 2. Basic monitoring equipment setup. $\mathrm{ET}=$ endotracheal tube; REC = recording electrodes; GND = ground electrodes; $\mathrm{EMG}=$ electromyography.

recommends that attention to initial standard equipment setup algorithm, including especially attention to proper endotracheal tube placement at the beginning of surgery, substantially reduces the overall monitoring problems encountered intraoperatively (Fig. 3).

\section{Algorithm for Monitoring Tube Placement Intubation}

Intubation is best achieved with a short-acting, nondepolarizing paralytic agent as noted previously. A tube size should be chosen that provides optimal tube contact with the vocal cords (typically \#7 for most adults). Lidocaine jelly and other tube lubricants should not be used on the monitoring endotracheal tube. Standard preformed monitoring endotracheal tubes currently are available in common sizes 6.0, 7.0, and 8.0. These endotracheal tubes have outer diameters that are slightly larger than standard similarly sized endotracheal tubes. The larynx should be intubated with the largest endotracheal tube considered safe, as this will optimize electrode contact with the vocal cords. This improves impedance (discussed later) and has not been met with any untoward laryngeal effect such as vocal cord or laryngeal injury or vocal cord granuloma in the several thousand patients so treated. The tube may be placed with or without a stylette. Pooled saliva may occur at the level of the vocal cords and may result in altered signal (see discussion of "salt bridging" in Standards in Intraoperative Loss of Signal Evaluation section). Preoperative use of a drying agent such as glycopyrrolate and intraoperative suction may be helpful in these circumstances. The degree of rotation (with respect to the right and left electrodes) of an endotracheal tube is a new parameter for anesthesia, and occasionally right-handed anesthesiologists tend to rotate the tube clockwise (often approximately $30^{\circ}$ ) inadvertently. Rotational error typically requires counterclockwise correction. A pen mark placed at 12 o'clock at the upper margin of the exposed electrodes can help to prevent rotational errors at intubation. Depth of insertion and degree of rotation of exposed electrodes relative to the vocal cords should be noted both by anesthesiologist and surgeon. Intubation may be through standard anesthesia laryngoscopes or with the use of newer video laryngoscopes,

\section{Equipment/Endotracheal Setup Standard}

\section{A. Endotracheal tube}

- Intubation - short-acting NMB, drying agent

- Electrodes at cords - note depth, no rotation, no salivary pooling

- Position patient - Then verify position via-Glottic Exam or - Respiratory Variation

-Then fix tube position

\section{B. Equipment}

- Ground electrodes - shoulder

- Monitor: $\quad-100 \mu \mathrm{V}$ event threshold -stimulation current 1-2 mA -impedance ( $<5$ kilo ohms per electrode)

- Separate monitor and electrocautery units

\section{Initial surgical field testing}

- $\quad$ Stimulate ipsilateral Vagus

Fig. 3. Equipment/endotracheal setup standard. NMB = neuromuscular block agent. 
which allow all operating room personnel to view the intubation and the final position of the endotracheal tube on a bedside monitor. Depth of insertion was found to be appropriate in an Asian population when the endotracheal tube was $20 \mathrm{~cm}$ measured at the corner of the mouth, a standard measurement for endotracheal tube depth. Men had slightly greater depth of insertion $(20.6 \pm 0.97 \mathrm{~cm}$ in men vs. $19.6 \pm 1.0 \mathrm{~cm}$ in women); their work and that of others have shown no significant relationship between endotracheal tube depth of insertion and height, age, weight, or body mass index. ${ }^{59,60}$ In this series, with great attention to initial tube position, the tube readjustment rate during the subsequent surgery was only $5.7 \%$.

\section{General Equipment Setup}

It is best to physically separate electrocautery units and monitoring units and to keep wires apart and untangled. Electrocautery units should be positioned more than 10 feet away from the neural monitoring unit. Electrocautery units, both monopolar and bipolar, may create electrical interference, which may be controlled through muting cables to temporarily silence the audio and visual functioning of the monitor during electrocautery unit discharge. Some newer EMG monitoring systems are able to monitor during bipolar electrocautery. One must keep in mind that the interfaceconnector box has a fuse that may be checked. From the monitor comes a stimulator probe, the sterile end of which resides on the operative field. After intubation, the stimulator probe is placed sterilely on the field, and its distal end is taken by the nonsterile assistant and plugged into the interface-connector box. The surgeon or a monitoring technician can observe and control the monitor. The monitor is the source of important visual information as well as the audio tones associated with EMG responses and so, in the absence of a monitoring technician, the surgeon should have visual access to the monitor to identify waveform characteristics. Monitoring is not affected by the activity of cardiac pacemakers and will not impact their functioning and is also compatible with both Harmonic and Ligasure technologies. In general, there is a minimal electrical interaction between the monitoring systems and surrounding operating room electrical circuitry.

\section{Recording and Stimulation Ground Electrodes}

The recording electrodes and stimulator electrode probe require grounding; small grounding electrodes are placed through adhesive or subdermal needle electrodes on the shoulder on the side of the monitor unit. The basic electrical setup of the endotracheal tube monitoring system is shown in Figure 2. The endotracheal tube electrodes and the grounding electrode for the endotracheal tube are plugged into the interface-connector box. Grounding electrodes for the endotracheal recording electrodes and for the stimulating probe may be placed on the sternum region if shoulder placement results in a particularly noisy baseline. The recording ground electrode is placed nearest to the surgical site, and the stimulator ground electrode is placed more distally to minimize stimulus artifacts.

\section{Patient Positioning and Tube Fixation}

After intubation, the patient is positioned for surgery in head extension. The thyroid bag and/or shoulder roll should be placed with the anesthesia staff carefully holding the endotracheal tube in position. After neck extension and patient positioning, the tube is secured in place with tape, and tube support is provided. If the tube is taped (and therefore fixed at the level of the mouth) before the patient is fully positioned (i.e., fully extended), patient positioning from neutral to extension can result in change of the endotracheal tube depth within the airway. If significant enough, this change in position can cause electrode malposition. Yap et al. found that the endotracheal tube may be displaced relative to a neutral intubating position up to $21 \mathrm{~mm}$ inward and up to $33 \mathrm{~mm}$ outward as the patient is moved into full neck extension, giving nearly $6 \mathrm{~cm}$ of possible endotracheal tube movement as the patient is taken from a neutral to a fully extended position. ${ }^{61}$ Other workers have also documented changes in endotracheal tube position during head and neck maneuvers in both adults and children. ${ }^{62,63}$ Therefore, all tests for adequate positioning in terms of endotracheal tube electrode vocal cord contact, must be obtained after the patient is fully extended. The tube should be taped in such a way that the tape can be easily removed should the tube need to be repositioned during the case. The tape should be applied at the level of the lips and not higher up on the tube as, this will tend to push the tube farther in during the case. After taping, attention should be given to ensure that the tube's position is stable and supported to prevent inward endotracheal tube displacement from the drapes or an assistant's arm resting on the tube. It is also important that the endotracheal tube and anesthesia circuit be supported such that rotational forces on the tube are limited during the case (such as a torque applied secondary to the attached anesthesia circuit).

\section{Preoperative Tube Position Verification Testing After Patient Positioning}

Since significant change in tube position may occur after intubation as the patient is taken from a neutral intubating position to extended position, the study group recommends that before surgery begins (after the patient is fully positioned), a tube-position verification test should be considered as a routine, certainly at the initial stages of a monitoring program and perhaps indefinitely. Such attention at this point in the case would obviate tube positional problems later in the case in a significant number of patients; this is supported by the work of Lu et al. ${ }^{59}$ There are two important points regarding tube-position verification. First, it is important that both anesthesiologist and surgeon jointly provide care in endotracheal tube verification; and second, these verification tests should be performed after 


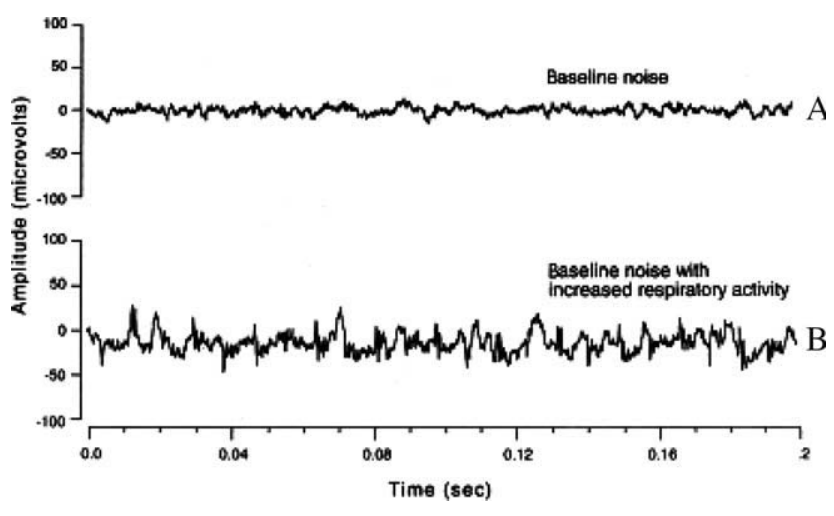

Fig. 4. Respiratory variation.

the patient is fully positioned, and not when the patient is in the neutral intubating position.

Limited data exists in the available literature in regard to such tube position verification tests. Adequate measures of impedance as read on the monitor imply only adequate recording electrode contact with the body, not necessarily correct vocal cord positioning. Two tube position options currently are available:

1. Respiratory variation. After intubation and after the paralytic agent from induction has worn off but before the inhalation plane of anesthesia is too deep, there is a window that occurs, typically just before the patient starts to move spontaneously or "buck." During this window, a coarsening of the monitor baseline can be seen, with small waveforms typically varying from 30 to $70 \mu \mathrm{V}$. This activity is termed "respiratory variation of the baseline" (Fig. 4). For this variation to be present on both channels, the endotracheal tube must be in good position at the level of the vocal cords. Impedance values alone, as noted previously, imply only good contact between the electrodes and the patient not necessarily at the level of the vocal cords. Good impedance and good respiratory variation before the patient's positioning are also not useful. However, the development of respiratory variation after the patient is completely positioned is associated with excellent tube positioning. The advantage of using respiratory variation as a tube verification test is that tube-positioning information is obtained without any instrumentation. However, in order to identify respiratory variation, the anesthesiologist must manage patients so they are light enough at the time of positioning to be able to see this activity and to be able to quickly sedate the patient once this is seen to prevent patient movement, or bucking. As soon as respiratory variation is seen on the monitor, an intravenous agent such as propofol should be given to sedate the patient quickly.

2. Repeat laryngoscopy. The anesthesiologist or surgeon may repeat visualization of the glottis after patient positioning. This visualization may be achieved with direct laryngoscopy or fiberoptic laryngoscopy. New video laryngoscopes are especially useful for such endotracheal position examinations. Alternately a fiber- optic scope may be applied to the endotracheal tube and be left indwelling during the case to provide ongoing information. This type of equipment setup is not routinely available and often will become malpositioned or obscured by saliva during the case, resulting in poor glottic-endotracheal tube visualization. Repeat laryngoscopy after patient positioning represents the most accurate method for tube positional assessment but does represent a separate procedure.

A "tap test" has been promoted as a tube-position verification test. However, data is not available to evaluate the accuracy of this method of endotracheal tube positioning localization. The test involves briskly tapping with a finger the midline larynx at the level of the thyroid cartilage or cricoid cartilage to determine whether there is a tap-induced response on the monitor. The physiology underlying the development of a response on the monitor in association with mechanical trauma to larynx is unclear. Nonetheless, it is true that a variety of different mechanical maneuvers focused on the larynx and thyroid at the beginning of thyroid surgery through the cervical skin can elicit a nerve-like response waveform. The mechanism is poorly understood and is felt to be a non-EMG artifactual event generated by small movement of the electrodes in the presence of small background magnetic fields. There was no support in the study group for the use of the tap test.

\section{Monitor Settings}

After all patient positioning is complete, monitor settings should be checked. Monitor assessment should include checking impedance values. These should be less than $5 \mathrm{k} \Omega$ for each electrode with an imbalance between electrodes of less than $1 \mathrm{k} \Omega$. If individual recording endotracheal tube electrode impedance is high, the electrode may be in poor contact with the patient, and the endotracheal tube should be readjusted to improve contact. Low individual electrode impedance (i.e., $<5 \mathrm{k} \Omega$ per electrode) suggests good electrode-patient contact. As noted previously, this does not necessarily mean the electrode is resting, as it should, against the vocal cord. High impedance imbalance also implies poor electrodepatient contact and the need for readjustment of the endotracheal tube. If all impedances are high, the ground electrodes should be replaced.

At this time, the monitor should be checked for an appropriate event threshold at $100 \mu \mathrm{V}$, and a stimulator probe should be set on a value of 1 to $2 \mathrm{~mA}$. If there has been a problem with false-positive stimulation, the event threshold can be turned up to $200 \mu \mathrm{V}$. With these settings, low-level respiratory spontaneous waveforms $<100 \mu \mathrm{V}$ will not trigger "evoked events" on the monitor (see respiratory variation of the baseline discussed earlier).

\section{Initial Testing on the Surgical Field}

Should there be any question as to tube position at the onset of surgery, two intraoperative options exist. The first is translaryngeal stimulation. Translaryngeal stimulation on the midline thyroid cartilage, cricothyroid membrane, 
and cricoid cartilage can be encoded to determine location within the larynx of exposed electrodes on the endotracheal tube. No data is available to evaluate the accuracy of this method of endotracheal tube positioning localization, although its application is intuitive. ${ }^{64}$ With this method, one must make sure to stimulate at a high enough current so as to optimally shunt current through the larynx. The surgeon should appreciate that the vocal cords are located approximately half way down the thyroid cartilage. If maximum shunt stimulation occurs at the level of the cricothyroid membrane, anterior arch of the cricoid, or lower, one assumes excessive endotracheal tube depth. The second option to determine tube position after surgery has begun is direct ipsilateral vagal nerve stimulation. With this simple technique, which is required for all patients at the outset of surgery in all cases (see later discussion), the finding of satisfactory EMG with vagal stimulation proves adequate endotracheal tube placement. These intraoperative tests of tube position (i.e., performed after the incision) imply that if the tube needs correction, it would have to be done under the drapes by the anesthesiologist and can be somewhat more cumbersome than if this information is obtained before the onset of surgery (see previously discussed tube verification tests).

At the onset of surgery, the larynx and the strap muscles are dissected. The stimulator probe can also be tested on the strap muscles to confirm gross muscle twitching, which assures lack of ongoing paralytic agent and intact stimulator function. When muscle in the surgical field is stimulated, the current distributed to the patient is recorded back on the monitor to confirm that that the correct level of stimulation has been delivered to the patient. When using the stimulator probe it is important to recognize that its output is pulsatile 4 per second. Therefore, it is important to drag the tip of the stimulator probe over tissue rather than to intermittently touch or "hop" over the tissue. Such stimulation may result in probe contact with tissue between pulses.

\section{Predissection Vagal Stimulation}

Before accepting any tissue as being truly negative (in terms of being the RLN) and to confirm overall system function, the surgeon visually identifies the vagus nerve and obtains a true-positive result. Only then can one be sure that the system is completely functional and that, as one searches for the RLN, one can trust a negative response. It is important for vagal stimulation to be the first and last step in each case (Fig. 3).

\section{STANDARDS IN INTRAOPERATIVE LOSS OF SIGNAL EVALUATION}

\section{Laryngeal Twitch Assessment: Present}

If the RLN is being stimulated and EMG activity is either not present or at unusually low amplitude below $100 \mu \mathrm{V}$, the first step should be assessment of the laryngeal twitch response by the surgeon, with vagal stimulation on that side (Fig. 5) ${ }^{65}$ If laryngeal twitch response is present, then the stimulation side of the monitoring system is working; that is, you are delivering current to a functional nerve-neural function is assured

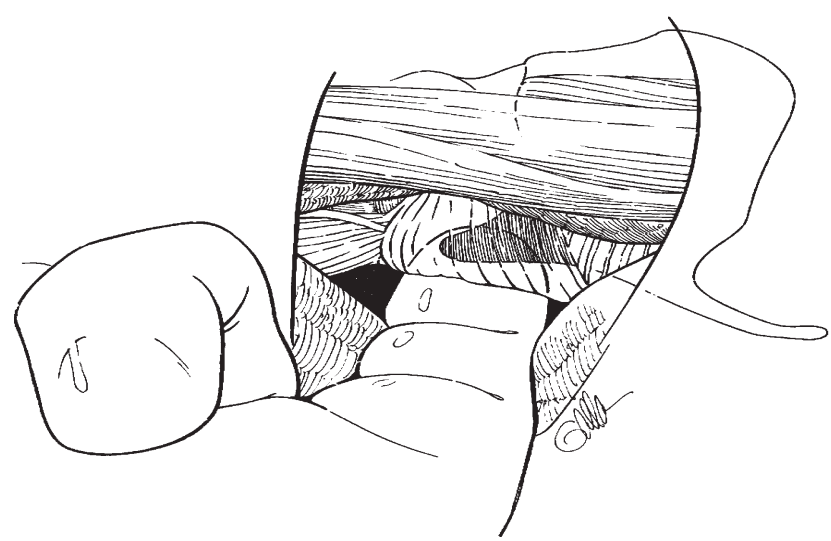

Fig. 5. Laryngeal twitch assessment.

and monitoring system dysfunction is present and this dysfunction is on the recording side (Fig. 2). In the vast majority of cases, recording-side dysfunction implies endotracheal tube electrode malposition. In this scenario, one should also consider the less likely possibility that the recording electrode ground is misplaced. Grounding electrodes can be dislodged or displaced through perspiration. Adhesive ground electrodes degrade with time and may not make good skin contact after their expiration date. Grounding electrodes are easily checked and repositioned. Recording-side electrodes and ground connections at the interface-connector box should also be checked in pursuit of a recording-side malfunction. A recent study attempting assessment of laryngeal twitch describes administration of neuromuscular blockage in study patients; this procedure, of course, precludes muscular response assessment. ${ }^{6}$ Other recent work suggests laryngeal twitch sensitively tracts with nerve function. ${ }^{65}$

\section{Contralateral Vagal Assessment}

An alternate troubleshooting algorithm, if an ipsilateral RLN stimulation is not giving adequate EMG signal, especially suited in cases of planned bilateral procedures is to dissect the contralateral vagus nerve. If the contralateral vagus also does not give good EMG, a recordingside (i.e., typically related to the endotracheal tube) problem is likely and is investigated as previously described. If the contralateral vagus nerve does stimulate normally (i.e., the endotracheal tube is therefore in good position), then there is, on the ipsilateral side, a stimulation error (including possible nerve injury) and is worked up accordingly (Fig. 6). Contralateral vagal stimulation therefore represents an option for troubleshooting in addition to laryngeal palpation, especially in planned bilateral procedures. One should note that certain tube-rotation scenarios could possibly give divergent stimulation parameters on the two separate channels and that this method necessitates contralateral strap muscle elevation, which may be a disadvantage if one ends up doing a staged secondary/completion procedure.

\section{Endotracheal Tube Assessment}

As noted previously, the most common recordingside problem is malposition of the endotracheal tube. 


\section{Intraoperative LOS Evaluation Standard}

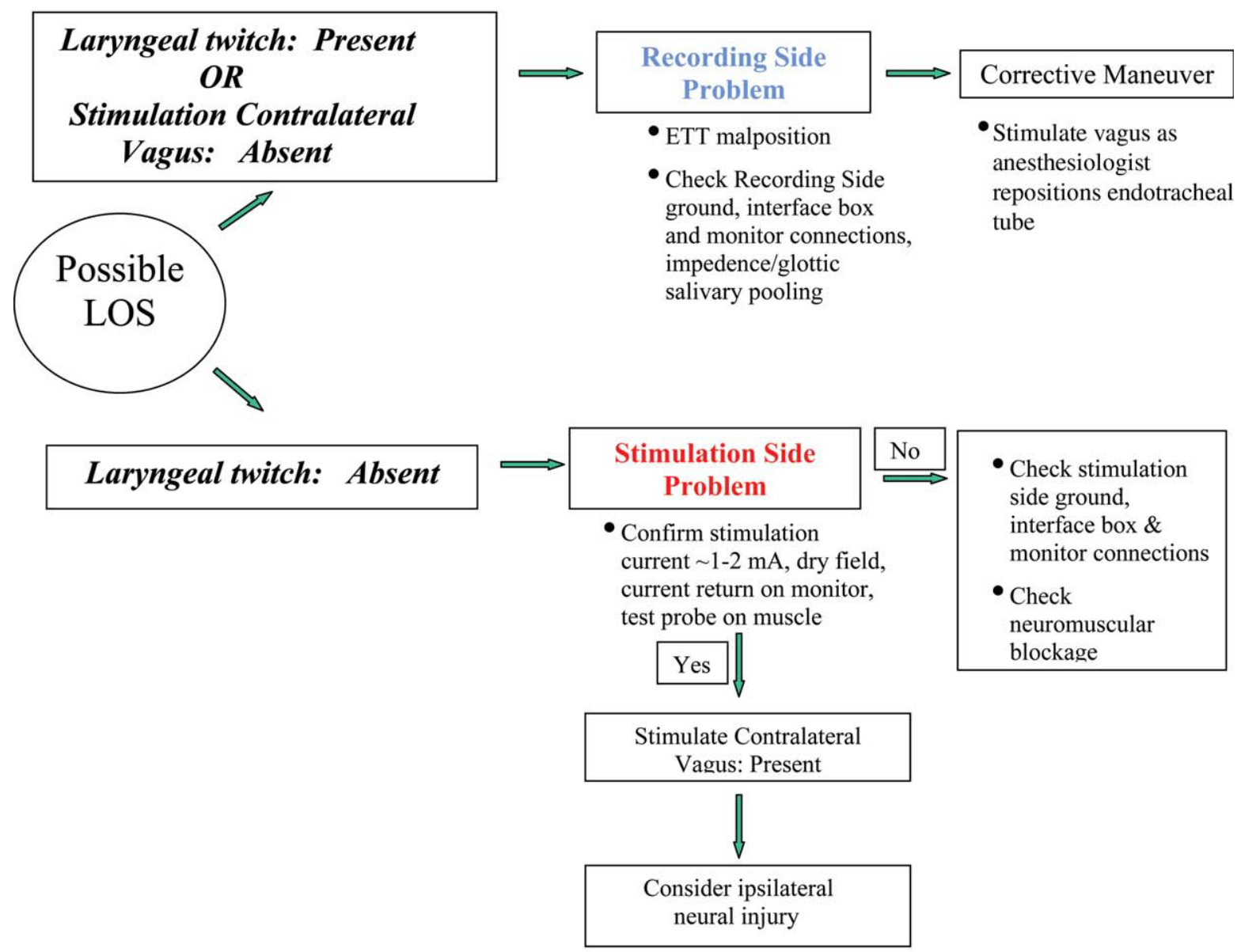

\section{LOS Definition:}

$1-E M G$ change from initial satisfactory $E M G$

2 -No or low response (i.e.100 $\mu$ V or less)with stimulation at 1-2 mA, dry field

3-No laryngeal twitch and/or observed glottic twitch

\section{With LOS:}

1-Map lesion and determine Type I(Segmental) or Type II (Global) injury
2-Consider contralateral surgery timing

Fig. 6. Intraoperative loss of signal evaluation standard. LOS = loss of signal; ETT = endotracheal tube; EMG = electromyography.

Malpositioned may imply either inadequate or excessive endotracheal tube depth relative to the vocal cords or endotracheal tube rotation. If patient has moved (through inadequate anesthesia at the beginning of the case) or if the larynx, trachea, or thyroid has been significantly manipulated, endotracheal tube position may change. This is true, for example, with extreme laryngeal/thyroid retraction during surgery and often may occur after delivery of a large substernal or cervical goiter. Any change in larynx position relative to the endotracheal tube may result in a malpositioned tube. $\mathrm{Lu}$ et al. ${ }^{59}$ showed that with attention to initial tube placement, optimal tube placement was possible in $94 \%$ of patients. In those $5.7 \%$ of patients requiring postintubation tube placement correction, 50\% required advancement of the endotracheal tube and 50\% required the endotracheal tube to be pulled back.

The corrective maneuver for endotracheal tube placement problems is vagal stimulation by the surgeon as the anesthesiologist readjusts the tube. This can be done empirically by the anesthesiologist based on the appearance of the endotracheal tube at the lips as compared to its appearance at the beginning of the case, or it can be done through direct visualization of the glottis through fiberoptic or direct laryngeal exam. This readjustment typically corrects the problem promptly. As soon 
as the anesthesiologist has established correct endotracheal tube placement, vagal stimulation results in robust waveform. This entire corrective maneuver with the anesthesiologist readjusting the tube and the surgeon stimulating the vagus nerve takes only a minute or two.

Endotracheal tube placement errors include also rotational errors. The electrical event underlying vocal cord depolarization and movement is a complex threedimensional electrical event. It appears based on experience with a number of different electrode designs that the posterior glottis is the more "electrically rich" area of the larynx as opposed to the anterior commissure. Rechecking monitor settings for correct impedance values may be helpful in detecting rotational displacement of the endotracheal tube. Some members of the study group have noticed that endotracheal tubes that are rotated relative to the normal position may result in more diffuse false-positive signals.

Another cause of endotracheal tube-associated problems can be the pooling of saliva at the level of the glottis. Although the exact mechanism is unclear, intraoperative observations suggest that serial impedance measures may increase during progressive salivary accumulation (perhaps through a "salt-bridging" phenomenon) and lead to gradual reduction in evoked responses during a case (unpublished observations, Lee Rea and Greg Randolph, 2010). As noted previously, intraoperative suction as well as the preoperative administration of a drying agent such as glycopyrrolate may be helpful.

\section{Laryngeal Twitch Assessment: Absent}

If laryngeal twitch is not present during neural stimulation, one must consider that a stimulation-side error (Fig. 2) has occurred and that stimulation current has not been effectively delivered. The stimulator probe can be checked on muscle to identify its twitch, and the monitor can be reviewed for appropriate current return. One must keep in mind that sufficient current must be applied to the RLN for it to depolarize. When the nerve is dissected free of fascia and completely dry, it will first give initial subthreshold depolarization at stimulation levels of 0.3 to $0.4 \mathrm{~mA}$ and give maximum depolarization at $0.8 \mathrm{~mA}$. One may consider probe malfunction if current delivery is not confirmed and may consider obtaining a new probe; one must also check stimulationside connections at the interface-connector box. Occasionally during cases in which the patient perspires, the ground skin electrodes may be dislodged.

If these issues with the stimulation-side system have been reviewed, one must consider whether the structure being stimulated is not nerve. In this scenario, the vagus is the safety net in that the vagus is so large and so easily indentified that it serves as a confirmation of neural anatomy and as a guaranteed bridge between visual neural anatomy and EMG stimulation response. Without vagal stimulation, one must now consider whether nerve neuromuscular blockage has been administered.

If stimulation of the RLN and vagus produce either no EMG activity (electrical silence) or substantially reduced EMG activity $(<100 \mu \mathrm{V})$ with absence of laryngeal twitch during stimulation and a careful point-by-point review of the LOS algorithm fails (Fig. 6) to detect any equipment problem or neuromuscular blockage, a surgeon must consider that this is true LOS and must strongly suspect neural injury. In the setting of injury, amplitude reduction should be associated with EMG response latency increase.

\section{Loss of Signal}

LOS, as noted previously, has yet to be accurately defined. However, the study group's experience with laryngeal EMG and the relationship between EMG evoked response and glottic function has allowed for some basic tenants in describing LOS. First, LOS can only be interpreted if the EMG signal was good initially (i.e., waveforms $>100 \mu \mathrm{V}$ ). If, from this satisfactory initial level of response, a signal degrades to $<100 \mu \mathrm{V}$ during reasonably robust suprathreshold level of stimulation (i.e., between 1 and $2 \mathrm{~mA}$ ), then LOS should be considered. Such isolated amplitude changes would be taken even more seriously if there were associated increased latency and increased threshold, as noted previously. Given the potential impact LOS may have on the surgical plan (i.e., aborting the second side surgery), the study group recommends adding either laryngeal twitch assessment or glottis observation to the previously noted EMG data. If these EMG data are present and there is either no laryngeal twitch or evoked glottis movement with stimulation, then the surgeon must consider this to be true LOS and that nerve injury has occurred. Of course, as noted previously, these data are interpreted in the setting of a robust and negative LOS equipment evaluation (Fig. 6).

With LOS, two issues should be considered: 1) identification of the site of lesion-that is, neural injury point mapping; and 2) consideration of optimal contralateral surgery timing.

In the setting of neural injury, during surgery the surgeon should endeavor to identify the segment of nerve injured starting from the most distal point of the RLN (i.e., at the laryngeal nerve entry site) stimulating from distal to proximal, serially testing the entire segment of nerve that has been dissected to see if a neuropraxic segment of signal loss can be identified. The identification of such a segment then can allow the surgeon to review the conduct of the surgery and potentially better understand the surgical maneuver that may have injured that specific segment, such as excessive traction, compression, or clamping. ${ }^{66}$ In the study group's experience, it appears nerve injuries identified through intraperative neural monitoring may be segregated into two basic types. The first involves a clearcut RLN segment that is lesioned, which we term type 1 RLN injury-segmental injury. One may be able to potentially correct the lesion if there is a clip or suture entrapping the nerve at this point and avoid permanent RLN injury. Such retrograde mapping of injury may show the nerve is, in all segments (entire RLN and vagus nerve), nonconductive. This implies a more global injury likely consistent with an intralaryngeal focus, which we term type 2 RLN injury-global injury. With LOS, one must consider that the ipsilateral nerve is injured at least temporarily, 
and so the surgeon can consider whether it is important and in the patient's best interest to perform surgery on the contralateral side on this day. With intraoperative LOS, the surgeon is empowered to avoid the potential of bilateral nerve paralysis. One may move forward with contralateral surgery when postoperative laryngeal exam confirms resolution of neuropraxia, typically in 6 to 8 weeks in many mild cases. With application of such a detailed LOS troubleshooting algorithm, the rate of negative signal at the end of surgery becomes reduced and the incidence of postoperative vocal cord paralysis in this group becomes substantially higher.

\section{Intraoperative RLN Stimulation Errors}

A variety of real and perceived stimulation errors may be experienced during RLN stimulation:

1. Ineffective intraoperative stimulation of the RLN:

- Paralytic agent on board or pseudo-cholinesterase deficiency $^{12}$

- Insufficient current delivery; blood and fascia covering the nerve or insufficient probe-nerve contact. Insufficient probe-nerve contact may also provide a false-negative response as stimulating current is pulsatile, and so the probe must be present on the nerve long enough to have a pulse stimulation applied to the nerve.

- Probe malfunction. Probe may be defective or probe wire or probe ground may not be connected appropriately.

- Equipment malfunctions, including most typically endotracheal tube positioning

- Monitor-event threshold set too high or monitor volume too low. Some monitors are set with stimulation artifact suppression. This silences any response that comes very early, near the stimulation artifact spike of delivered current. The purpose of the stimulation artifact suppression is to silence the tail end of stimulation artifact so that it is not recorded as an evoked response. When stimulating the distal segments of the RLN, the latency may be very short and the evoked response (or part of it) may occur within this stimulus suppression artifact period and be falsely suppressed. Monitors may be adjusted to shorten the stimulation artifact suppression.

- Insufficient stimulator current. This can be an important cause of false-negative responses. Generally suprathreshold stimulation is optimal at $2 \mathrm{~mA}$ when looking for the nerve, and $1 \mathrm{~mA}$ after finding it is the proper stimulation level for routine monitoring.

2. Intraoperative nonneural shunt stimulation:

- Stimulation very near with current spread to the nerve. This may be more common at high (i.e. $2 \mathrm{~mA}$ ) than at $1 \mathrm{~mA}$. Blood or blood vessel may shunt current from nonneural to neural tissue. This seems especially common with small or medium-sized arterial branches that bridge or cross the nerve. In these scenarios it is best to turn down the stimulation current to a level where false-positive stimulation is silenced. It is important to make sure that the nerve stimulates robustly at this lower stimulating current. This rarely requires turning current below 0.5 to $0.8 \mathrm{~mA}$. Another option with diffusely positive stimulation is to turn the monitor event threshold up from 100 to $200 \mu \mathrm{V}$.

- Transtracheal stimulation; the shunting of current directly to the electrodes. Generally the surgeon would know if he is stimulating the trachea, but an RLN (especially in the left paratracheal region or at the ligament of Berry) may be adjacent to the trachea and lead to this error.

3. Various anomalous responses to RLN stimulation related to either the recording or stimulation side, or both:

- Simultaneous use of electrocautery surgical instrument prohibits stimulator use. For the duration of use of electrocautery, muting cables are provided to disable auditory and visual monitoring. Some newer EMG monitoring systems are not muted and are able to monitor during bipolar electrocautery. External sources including cell phones, diathermy, electrocautery used in the operating room or adjacent operating rooms, or other sources of electromagnetic interference may induce recording muting.

- Salivary pooling at the level of the glottis. Administration of a drying agent at the onset of the case and suctioning may minimize this effect, which may be associated with increased endotracheal electrode impedance.

- Shorted-out EMG electrodes (for example if they touch left to right) or monitor or interface-connector box fuse blown. Neural monitoring equipment including interface-connector box should have adequate separation from the wires of the electrosurgical unit.

- False-positive findings during nerve stimulation may be more common in the setting of excessive tube rotation.

- False responses may occur when two metal instruments strike together within the surgical field, such as a metal instrument and a metal suction tube (Fig. 7). Such signals are typically shifted to the right relative to stimulation artifact and are sharp peaked monophasic waves with fast onset and offset.

- False-positive activity may occur when recording electrodes and stimulator cables become tangled, resulting in stimulus artifact being spuriously detected as an EMG event. Such artifact waveforms are substantially different in appearance from the standard evoked EMG waveform, which is typically biphasic or triphasic and timed relative to the stimulation artifact (Fig. 7). However, these artifacts may result in a falsepositive tone from the monitor. Inadvertent 


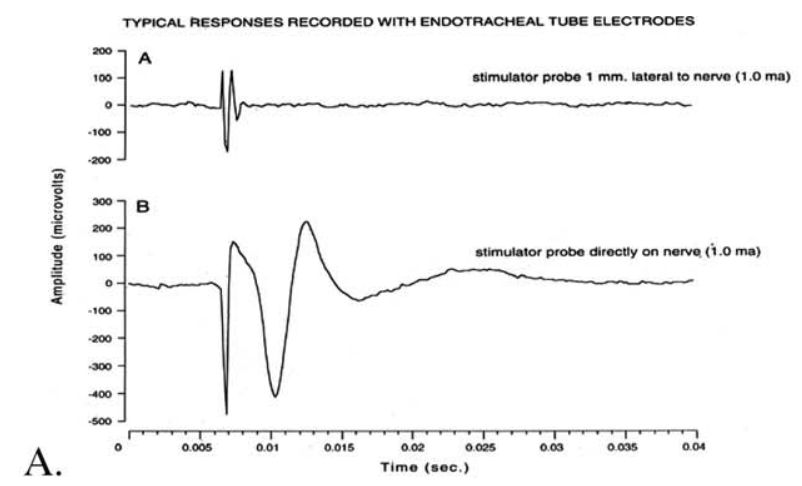

B.

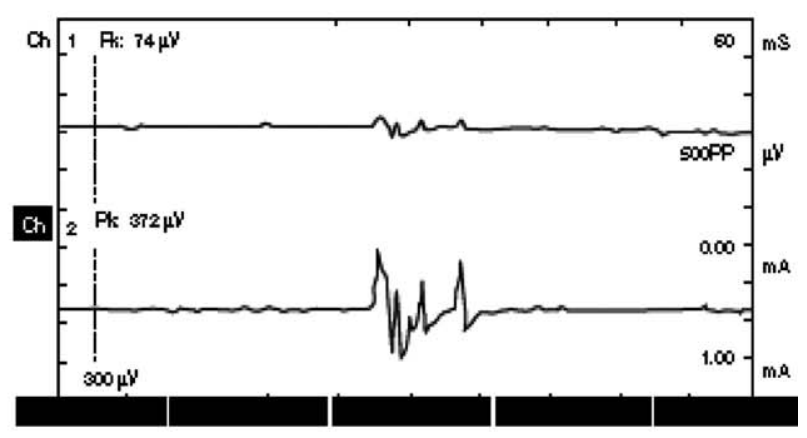

Fig. 7. (A) Stimulation artifact (upper panel) and normal recurrent laryngeal nerve waveform (lower panel). (B) Metal-on-metal artifact. RLN = recurrent laryngeal nerve.

manipulation of electrode wires of patient connectorinterface box or cable or use of the handheld electrical stimulator by the anesthesiologist may result in falsepositive signals.

- Phasic false-positive activity coincident with respiration may occur when the endotracheal tube cuff deflates enough to allow ventilated air to move retrograde and reflux through the glottis.

- Cold irrigation, heat from adjacent prolonged use of bipolar retraction on the RLN and patients who are in light planes of anesthesia may be associated with a spontaneous continuous train of EMG response from a nerve that has not been directly stimulated with the stimulator.

\section{STANDARDS IN WAVEFORM DEFINITION AND ASSESSMENT}

Standard definitions and terminology that exist for laryngeal EMG can be applied to intraoperative nerve monitoring EMG. ${ }^{67}$ Normative amplitude, latency, and threshold data for RLN and vagal intraoperative stimulation are just now being initially defined, in contrast to evoked potentials in other clinical neurophysiology applications. ${ }^{68}$ The basic evoked waveform for the human RLN or vagus nerve is typically biphasic or triphasic (Fig. 7).

\section{Amplitude}

Amplitude of the evoked response through stimulation of the vagal and RLN has not been uniformly defined within the surgical monitoring literature. The typically biphasic waveform represents the summated motor action unit potentials of the ipsilateral vocal cord muscle. Measures of amplitude may be correlated with the number of muscle fibers participating in the polarization during standard laryngeal EMG. Vocal cord depolarization amplitudes range from 100 to $800 \mu \mathrm{V}$ during normal awake volitional speech. ${ }^{67}$

Using existing standards in EMG monitoring physiology, we define monitoring waveform amplitude as the height from the vertical height of the apex of the positive initial waveform deflection to the lowest point in the next subsequent opposite polarity phase of the waveform (i.e., peak to peak). Amplitudes during intraoperative monitoring may vary significantly within a patient and among patients. Amplitude may vary during intraoperative nerve monitoring because of variations in several factors: 1) Variation in the degree to which the field is affected with fluid or blood, 2) variation in degree of probe-nerve contact during stimulation and variation in degree to which the stimulated nerve is ensheathed in fascia, 3) variation in environmental temperature or in irrigation fluid used, and 4) variation in recording electrode surface endotracheal tube position.

\section{Threshold}

Threshold is defined as the current that, applied to the nerve, first starts to trigger minimal EMG activity. In humans the RLN and vagus nerve will first begin to stimulate at approximately 0.3 to $0.4 \mathrm{~mA}$ if the nerve is dry and dissected free of fascia. ${ }^{28}$ The response amplitude that results at threshold stimulation is lower than the maximum amplitude achieved as stimulation current increases toward $0.8 \mathrm{~mA}$. At maximum stimulation, all nerve fibers are being depolarized and maximal stimulation is achieved. Beyond this point, increasing stimulating current does not lead to further increases in recorded EMG. This is the rationale for stimulation during the bulk of the case at $1 \mathrm{~mA}$, which represents a good and safe suprathreshold stimulation. The use of 2 mA does not get any higher EMG amplitude but depolarizes a greater sphere of tissue around the probe tip and so has utility when initial searching/mapping out the RLN.

\section{Latency}

Latency has not been uniformly defined within the surgical monitoring literature. Whereas amplitude is believed generally to represent the number of fibers participating in the depolarization event, latency has generally been believed to be associated with the speed or ease of stimulation-induced depolarization and depends on the distance of the stimulation point to the ipsilateral vocal cord. Given the different length of the vagus nerve on both sides, latency is significantly longer at the left compared to the right side when the vagus is 
stimulated in the midneck during thyroidectomy. Although no standard exists as to the exact point on the waveform that is best used for calculating absolute latency, Schwartz and Berry note that "prevailing opinion suggests that latency is measured at a point representing the beginning of the down-slope of a given peak component." ${ }^{\circ 9}$ Using existing standards in neural monitoring physiology and in auditory evoked response testing, we therefore may define latency as the time from the stimulation spike to the first evoked waveform peak. Measuring latency to the first evoked waveform deflection from the zero baseline is a much more variable measure and requires agreement, with each measurement, as to exactly where the waveform first leaves the baseline. Recent unpublished normative data suggest mean RLN latency (with nerve stimulation within the surgical thyroid bed) is 3.97 milliseconds, and superior laryngeal nerve latency (when stimulated at the level of the thyroid cartilage) is 3.5 milliseconds. Right vagus latency (when stimulated at the level of the thyroid cartilage) is 5.4 milliseconds, and left vagus latency is 8.1 milliseconds (unpublished data, Randolph and Dralle, 2010). Latencies during intraoperative monitoring are discrete enough to distinguish artifacts from neural stimulated structures and to differentiate RLN, superior laryngeal nerve, and vagus nerve and within vagal stimulation, to distinguish left from right vagus easily.

\section{End of Surgery Neural Testing and Prognostica- tion of Vocal Cord Function}

The study group agreed that intraoperative EMG (vs. audio tone alone) in combination with postoperative vocal cord mobility should be the basis for test definition. Using postoperative vocal cord mobility as the main outcome parameter for RLN function, a true-negative test is defined by the existence of a typical vocal cord EMG response at the end of the procedure with intact vocal cord mobility postoperatively. In contrast, if there is at the end of surgery a LOS combined with postoperative vocal cord paralysis, that outcome is defined as a true-positive test.

False positives (i.e. LOS with intact vocal cord mobility). Causes of false positives include:

1. Various equipment problems both on stimulation side and on recording side, most commonly endotracheal tube displacement

2. Blood or fascia covering the stimulated nerve segment

3. Neuromuscular blockage

4. Early response elimination due to the stimulation suppression artifact caught off segment

5. Vocal cord paralysis early neural recovery. There is some evidence from larger IONM series that transient neuropraxia may be of short duration. There has been experience within the study group of transient LOS with regain of signal before the end of surgery ${ }^{12}$ (Randolph, unpublished communications, 2010). It seems likely that with time we will document neuropraxic states lasting seconds, minutes, or days as well as more typical patients with several weeks to several months of transient dysfunction. The earlier our postoperative glottis exam, the more of these very acute transient neuropraxic patients we will likely see.

\section{False negatives (i.e. good EMG with postopera-} tive vocal cord paralysis). Under physiological conditions, normal vocal fold mobility correlates with positive motor unit action (MUAP) activation, but the evoked stimulation of the vagus or RLN at the completion of surgery with a stimulating probe at $1 \mathrm{~mA}$ is a nonphysiologic event. As Koester et al. note, "Positive MUAP activation can only imply that there is at least partial continuity of the RLN-thyroarytenoid muscle axis. It does not guarantee adequacy of power, contraction or elimination of fatigue in a partially denervated or deconditioned thyroarytenoid muscle."70 Although the relationship of intraoperative nonphysiologic nerve stimulation and postoperative volitional function is not completely understood, it does appear that when the signal is reasonably robust intraoperatively, the correlation with postoperative volitional function is excellent such that negative predictive value is more than $95 \%$, as seen several recent series. ${ }^{6,7,17,21,22,25,26}$ In the study group's experience, occurrences of vocal cord paralysis when intraoperative testing is normal have all been uniformly temporary, often without severe symptoms.

Causes of false negatives include:

1. Distal stimulation relative to injured nerve segment. This is the rationale for vagal stimulation for end-ofsurgery neural function prognosis.

2. Injury subsequent to last testing stimulation

3. Delayed neuropraxia. One may hypothesize progressive edema, which might perhaps impact on the RLN at an intralaryngeal location at the cricothyroid joint articulation or alternately a delayed vascular effect.

4. Posterior branch injury. Endotracheal tube monitoring systems assess only the vocal cord muscle, not the PCA muscle. Separate posterior cricoid muscle electrodes are required for monitoring the PCA branch of the RLN. It is conceivable, but not likely, that in a given patient there are extra laryngeal branches that reveal a posterior branch with PCA fibers in which discrete injury to the posterior branch might occur with ongoing good positive signal in the anterior thyroartenoid-vocalis muscle branch and which may be revealed postoperatively as a complete defect of abduction. ${ }^{49,50}$

5. Vocal cord immobility due to nonsurgical issues such as hemi-laryngeal edema or arytenoid cartilage dislocation

\section{Prognostication Statistics}

Concerning permanent vocal cord function, Dralle recently reviewed six studies showing that the negative predictive value varied from $92 \%$ to $100 \%$, but the positive predictive value varied greatly from $10 \%$ to $90 \%{ }^{26}$ The average in six studies reviewed by Dralle of positive predictive value is only 45\%..$^{6,7,21-23,25}$ Aggressive use of the endotracheal tube placement algorithm and 
troubleshooting algorithm has been shown to increase the positive predictive value from $11.6 \%{ }^{21}$ to $75 \%$ or higher (Randolph, unpublished observation, 2010).

\section{Documentation}

EMG signals may be recorded and printed out to be filed for future reference. Documentation of neural physiologic signals may be applied to forensic and long-term studies of vocal cord outcome. There is no uniform agreement as to the formatting of waveform documentation. The study group suggests if documentation is required for thyroidectomy, an initial timed recorded waveform including measures of amplitude, latency, waveform morphology, and magnitude of stimulating current be measured at the beginning, during, and completion of surgery for ipsilateral RLN (stimulated at the level of the thyroid bed) and vagus nerve (stimulated at the level of the thyroid cartilage).

\section{BIBLIOGRAPHY}

1. Horne SK, Gal TJ, Brennan JA. Prevalence and patterns of intraoperative nerve monitoring for thyroidectomy. Otolaryngol Head Neck Surg 2007; 136:952-956

2. Sturgeon C, Sturgeon T, Angelos P. Neuromonitoring in thyroid surgery: attitudes, usage patterns, and predictors of use among endocrine surgeons. World J Surg 2009;33:417-425.

3. Eltzschig HK, Posner M, Moore FD Jr. The use of readily available equipment in a simple method for intraoperative monitoring of recurrent laryngeal nerve function during thyroid surgery: initial experience with more than 300 cases. Arch Surg 2002;137:452-456; discussion 456-457.

4. Gavilan J, Gavilan C. Recurrent laryngeal nerve: identification during thyroid and parathyroid surgery. Arch Otolaryngol Head Neck Surg 1986;112:1286-1288.

5. Echeverri A, Flexon PB. Electrophysiologic nerve stimulation for identifying the recurrent laryngeal nerve in thyroid surgery: review of 70 consecutive thyroid surgeries. Am Surg 1998;64:328-333.

6. Tomoda C, Hirokawa Y, Uruno T, et al. Sensitivity and specificity of intraoperative recurrent laryngeal nerve stimulation test for predicting vocal cord palsy after thyroid surgery. World J Surg 2006;30:1230-1233.

7. Hamelmann WH, Meyer T, Timm S, Timmermann W. A critical estimation of intraoperative neuromonitoring (IONM) in thyroid surgery. Zentralbl Chir 2002;127:409-413.

8. Kunath M, Marusch F, Horschig P, Gastinger I. The value of intraoperative neuromonitoring in thyroid surgery: a prospective observational study with 926 patients. Zentralbl Chir 2003;128:187-190.

9. Jonas J, Bahr R. Intraoperative neuromonitoring of the recurrent laryngeal nerve: results and learning curve. Zentralbl Chir 2006;131: $443-448$

10. Hemmerling TM, Schmidt J, Bosert C, Jacobi KE, Klein P. Intraoperative monitoring of the recurrent laryngeal nerve in 151 consecutive patients undergoing thyroid surgery. Anesth Analg 2001;93:396-399.

11. Dackiw AP, Rotstein LE, Clark OH. Computer-assisted evoked electromyography with stimulating surgical instruments for recurrent/external laryngeal nerve identification and preservation in thyroid and parathyroid operation. Surgery 2002;132:1100-1106; discussion 1107-1108.

12. Snyder S, Hendricks J. Introperative neurophysiology testing of the RLN plaudits and pitfalls. Surgery 2005;138:1183-1192.

13. Brauckhoff M, Walls G, Brauckhoff K, Thanh PN, Thomusch O, Dralle H. Identification of the non-recurrent inferior laryngeal nerve using intraoperative neurostimulation. Langenbecks Arch Surg 2002;386:482-487.

14. Yarbrough DE, Thompson GB, Kasperbauer JL, Harper CM, Grant CS. Intraoperative electromyographic monitoring of the recurrent laryngeal nerve in reoperative thyroid and parathyroid surgery. Surgery 2004;136: $1107-1115$

15. Robertson ML, Steward DL, Gluckman JL, Welge J. Continuous laryngeal nerve integrity monitoring during thyroidectomy: does it reduce risk of injury? Otolaryngol Head Neck Surg 2004;131:596-600.

16. Dralle H, Sekulla C, Haerting J, et al. Risk factors of paralysis and functional outcome after recurrent laryngeal nerve monitoring in thyroid surgery. Surgery 2004;136:1310-1322.

17. Chan WF, Lang BH, Lo CY. The role of intraoperative neuromonitoring of recurrent laryngeal nerve during thyroidectomy: a comparative study on 1000 nerves at risk. Surgery 2006;140:866-872.discussion 872-873.

18. Shindo M, Chheda NN. Incidence of vocal cord paralysis with and withou recurrent laryngeal nerve monitoring during thyroidectomy. Arch Otolaryngol Head Neck Surg 2007;133:481-485.
19. Barczynski M, Konturek A, Cichon S. Randomized clinical trial of visualization versus neuromonitoring of recurrent laryngeal nerves during thyroidectomy. Br J Surg 2009;96:240-246.

20. Timmerman W, Hammelamnn W. Thyroid surgery: neuromonitoring of the RLN during thyroid surgery. Dtsch Arztebl 2004;101:7.

21. Thomusch O, Sekulla C, Machens A, Neumann HJ, Timmermann W, Dralle H. Validity of intraoperative neuromonitoring signals in thyroid surgery. Langenbecks Arch Surg 2004;389:499-503.

22. Beldi G, Kinsbergen T, Schlumpf R. Evaluation of intraoperative recurrent nerve monitoring in thyroid surgery. World $J$ Surg 2004;28:589-591.

23. Chan WF, Lo CY. Pitfalls of intraoperative neuromonitoring for predicting postoperative recurrent laryngeal nerve function during thyroidectomy. World J Surg 2006;30:806-812.

24. Dionigi G, Bacuzzi A, Boni L, Rovera F, Dionigi R. What is the learning curve for intraoperative neuromonitoring in thyroid surgery? Int $J$ Surg 2008;6(suppl 1):S7-S12.

25. Hermann M, Hellebart C, Freissmuth M. Neuromonitoring in thyroid surgery: prospective evaluation of intraoperative electrophysiological responses for the prediction of recurrent laryngeal nerve injury. Ann Surg 2004;240:9-17.

26. Dralle H, Sekulla C, Lorenz K, Brauckhoff M, Machens A. German IONM Study Group: Intraoperative monitoring of the recurrent laryngeal nerve and thyroid surgery. World J Surg 2008;32:1358-1366.

27. White WM, Randolph GW, Hartnick CJ, Cunningham MJ. Recurrent laryngeal nerve monitoring during thyroidectomy and related cervical procedures in the pediatric population. Arch Otolaryngol Head Neck Surg 2009; 135:88-94.

28. Randolph GW. Surgical anatomy of the recurrent laryngeal nerve. In: Randolph GW, ed. Surgery of the Thyroid and Parathyroid Glands. Philadelphia, PA: Saunders; 2003: 300-349.

29. Mihai R, Randolph, GW. Thyroid surgery, voice and the laryngeal examination-time for increased awareness and accurate evaluation. World $J$ Endocr Surg 2009;1:1-5.

30. Randolph GW, Kamani D. The importance of preoperative laryngoscopy in patients undergoing thyroidectomy: voice, vocal cord function, and the preoperative detection of invasive thyroid malignancy. Surgery 2006; 139:357-362.

31. James AG, Crocker S, Woltering E, Ferrara J, Farrar W. A simple method for identifying and testing the recurrent laryngeal nerve. Surg Gynecol Obstet 1985;161:185-186.

32. Otto RA, Cochran CS. Sensitivity and specificity of intraoperative recurrent laryngeal nerve stimulation in predicting postoperative nerve paralysis. Ann Otol Rhinol Laryngol 2002;111:1005-1007.

33. Riddell V. Thyroidectomy: prevention of bilateral recurrent nerve palsy: results of identification of the nerve over 23 consecutive years (1946-69) with a description of an additional safety measure. Br J Surg 1970;57: $1-11$.

34. Premachandra DJ, Radcliffe GJ, Stearns MP. Intraoperative identification of the recurrent laryngeal nerve and demonstration of its function. $L a-$ ryngoscope 1990;100:94-96.

35. Scheuller MC, Ellison D. Laryngeal mask anesthesia with intraoperative laryngoscopy for identification of the recurrent laryngeal nerve during thyroidectomy. Laryngoscope 2002;112:1594-1597.

36. Woltering EA, Dumond D, Ferrara J, Farrar WB, James AG. A method for intraoperative identification of the recurrent laryngeal nerve. Am $J$ Surg 1984;148:438-440.

37. Lipton RJ, McCaffrey TV, Litchy WJ. Intraoperative electrophysiologic monitoring of laryngeal muscle during thyroid surgery. Laryngoscope 1988;98:1292-1296.

38. Rice DH, Cone-Wesson B. Intraoperative recurrent laryngeal nerve monitoring. Otolaryngol Head Neck Surg 1991;105:372-375.

39. Tschopp K, Probst R. New aspects in surgery of the thyroid gland with intraoperative monitoring of the recurrent laryngeal nerve. Laryngorhinootologie 1994;73:568-572.

40. Jonas J, Bahr R. Neuromonitoring of the external branch of the superior laryngeal nerve during thyroid surgery. Am J Surg 2000;179:234-236.

41. Tschopp KP, Gottardo C. Comparison of various methods of electromyographic monitoring of the recurrent laryngeal nerve in thyroid surgery. Ann Otol Rhinol Laryngol 2002;111:811-816.

42. Eisele DW. Intraoperative electrophysiologic monitoring of the recurrent laryngeal nerve. Laryngoscope 1996;106:443-449.

43. Barwell J, Lytle J, Page R, Wilkins D. The NIM-2 nerve integrity monitor in thyroid and parathyroid surgery. Br J Surg 1997:84:854.

44. Horn D, Rotzscher VM. Intraoperative electromyogram monitoring of the recurrent laryngeal nerve: experience with an intralaryngeal surface electrode. A method to reduce the risk of recurrent laryngeal nerve injury during thyroid surgery. Langenbecks Arch Surg 1999;384: 392-395.

45. Timon CI, Rafferty M. Nerve monitoring in thyroid surgery: is it worthwhile? Clin Otolaryngol Allied Sci 1999;24:487-490.

46. Lamade W, Meyding-Lamade U, Buchhold C, et al. First continuous nerve monitoring in thyroid gland surgery. Chirurg 2000;71:551-557.

47. Hemmerling TM, Schmidt J, Wolf T, Wolf SR, Jacobi KE. Surface vs intramuscular laryngeal electromyography. Can J Anaesth 2000;47:860-865.

48. Brennan J, Moore EJ, Shuler KJ. Prospective analysis of the efficacy of continuous intraoperative nerve monitoring during thyroidectomy, parathyroidectomy, and parotidectomy. Otolaryngol Head Neck Surg 2001; 124:537-543. 
49. Rea L, Kahn A. Clinical evoked electromyography for current laryngeal nerve preservation: use of an endotracheal tube electrode in a postcricoid surface electrode. Laryngoscope 1998;108:1418-1420.

50. Marcus B, Edwards B, Yoo S, et al. Recurrent laryngeal nerve monitoring in thyroid and parathyroid surgery: the University of Michigan experience. Laryngoscope 2003;113:356-361.

51. Scott AR, Chong PS, Hartnick CJ, Randolph GW. Spontaneous and evoked laryngeal electromyography of the thyroarytenoid muscle: a canine model for intraoperative recurrent laryngeal nerve monitoring. Ann Rhinol Laryngol 2010;119:54-63.

52. Scott AR, Chong PS, Brigger M, Randolph GW, Hartnick CJ. Serial electromyography of the thyroarytenoid muscles in a canine model of vocal fold paralysis. Ann Otol Rhinol Laryngol 2009;118:56-66.

53. Sloan TB, Erian R. Effect of atracurium-induced neuromuscular block on cortical motor-evoked potentials. Anesth Analg 1993;76:979-984.

54. Sloan TB. Anesthesia and intraoperative neurophysiological monitoring in children. Childs Nerv Syst 2010;26:227-235.

55. Marusch F, Hussock J, Haring G, Hachenberg T, Gastinger I. Influence of muscle relaxation on neuromonitoring of the recurrent laryngeal nerve during thyroid surgery. Br J Anaesth 2005;5:596-600.

56. Bragg P, Fisher DM, Shi J, Donati F, Meistelman C, Lau M, Sheiner LB Comparison of twitch depression of the adductor pollicis and the respiratory muscles. Anesthesiology 1994;80:310-319.

57. Meistelman C. Effects on laryngeal muscles and intubating conditions with new generation muscles relaxants. Acta Anaesthesiol Belg 1997;48: $11-14$.

58. Chu KS, Wu SH, Lu IC, et al. Feasibility of intraoperative neuromonitoring during thyroid surgery after administration of nondepolarizing neuromuscular blocking agents. World $J$ Surg 2009;33:1408-1413.

59. Lu IC, Chu KS, Tsai CJ, et al. Optimal depth of NIM EMG endotracheal tube for IONM of the RLN during thyroidectomy. World J Surg 2008;32: 1935-1939.
60. Cherng $\mathrm{CH}$, Wong CS, Hsu CH, Ho ST. Airway length in adults: estimation of the optimal endotracheal for orotracheal intubation. Clin Anesthes 2002;14:271-274.

61. Yap SJ, Morris RW, Pybus DA. Alterations in endotracheal tube position during general anesthesia. Anaesthesia Intensive Care 1994;22:586-588.

62. Kim JH, Hong DM, Oh AY, Han SH. Tracheal shortening during laparoscopic gynecologic surgery. Acta Anesthesiol Scand 2007;51:235-238.

63. Jin-Hee K, Ro YJ, Seong-Won M, et al. Elongation of the trachea during neck extension in children: implications of the safety of endotracheal tubes. Anesth Analg 2005;101:974-977.

64. Chiang FY, Lu IC, Kuo WR, Lee KW, Chang NC, Wu CW. The mechanism of recurrent laryngeal nerve injury during thyroid surgery: the application of intraoperative neuromonitoring. Surgery 2008;143:743-749.

65. Randolph GW, Kobler JB, Wilkins J. Recurrent laryngeal nerve identification during thyroid surgery: laryngeal palpation. World J Surg 2004;28: $755-760$.

66. Snyder SK, Lairmore TC, Hendricks JC, Roberts JW. Elucidating mechanisms of recurrent laryngeal nerve injury during thyroidectomy and parathyroidectomy. J Am Coll Surg 2008;206:123-130.

67. Blitzer A, Crumley RL, Dailey SH, et al. Recommendations of the Neurolaryngology Study Group on laryngeal electromyography. Otolaryngol Head Neck Surg 2009;140:782-793.

68. ACNS guidelines for evoked potentials 9A. Available at: http://www.ACNS org. Accessed February 10, 2006

69. Schwartz DM, Berry GA. Normative aspects of the ABR. In: Jacobson JT, ed. The Auditory Brainstem Response. San Diego, CA: College Hill Press; 1985:65-97.

70. Koester M, Zorowka P, Wolf S. Neuromonitoring in thyroid surgery: the view of the laryngologist. Eur Surg 2003;35:246-249. 